\title{
Estudio comparativo de opinión de adolescentes sobre Educación Sexual en instituciones públicas y privadas
}

\section{Comparative study of adolescent opinion on Sex Education in public and private institutions} PACHECO-ARENAS, Noelia*†, MERCADO-MOJICA, Alin Jannet y HERNÁDEZ-MAQUEDA,
Martha Soledad

Universidad Veracruzana, facultad de Trabajo Social, calle 12 No. 215 Colonia Cazones, Poza Rica, Ver.

ID $1^{\text {er }}$ Autor: Noelia, Pacheco-Arenas / ORC ID: 0000-0002-7021-9564, CVU CONACYT ID: 391351

ID $1^{\mathrm{er}}$ Coautor: Alin Jannet, Mercado- Mojica / ORC ID: 0000-0002-7174-1573, CVU CONACYT ID: 473347

ID $2^{\text {do }}$ Coautor: Martha Soledad, Hernández- Maqueda / ORC ID: 0000-0001-8487-7931, CVU CONACYT ID: 514914

DOI: $10.35429 / J B E .2019 .8 .3 .9 .21$

Recibido 05 de Abril, 2019; Aceptado 25 de Junio, 2019

\section{Resumen}

En México los adolescentes reciben educación sexual desde la educación básica, sin embargo no todos los adolescentes comprenden los conocimientos que necesitan saber para tomar decisiones en relación a su vida sexual, por lo que el índice de embarazos no deseados e infecciones de transmisión sexual (ITS) continúa en ascenso. Ante este escenario las Trabajadoras Sociales como otros profesionistas, debemos asumir el compromiso de implementar acciones de educación sexual para la prevención del embarazo a temprana edad, la salud sexual y reproductiva y la promoción de los derechos sexuales, por lo que surge el interés de realizar un estudio comparativo sobre la opinión de adolescentes de dos instituciones educativas públicas y dos privadas de la Ciudad de Poza Rica, Veracruz, sobre su educación sexual. En el estudio se presentan resultados obtenidos a través la aplicación de un instrumento conformado por 29 interrogantes enfocadas en conocer la opinión de los adolescentes sobre su educación sexual, métodos anticonceptivos y embarazo en la adolescencia. El objetivo de la investigación es conocer la perspectiva de los estudiantes de dos instituciones educativas privadas y dos públicas sobre educación sexual y embarazo adolescente, para establecer propuestas sobre una Educación Sexual integral.

Educación sexual, Adolescentes, Trabajo Social

\begin{abstract}
In Mexico, adolescents receive sexual education from basic education, however, not all adolescents understand the knowledge they need to know to make decisions in relation to their sexual life, so the rate of unwanted pregnancies and sexually transmitted infections (STIs)) continues to rise. Given this scenario, Social Workers, like other professionals, must assume the commitment to implement sexual education actions for the prevention of pregnancy at an early age, sexual and reproductive health and the promotion of sexual rights, so that the interest of carrying out a comparative study on the opinion of adolescents from two public and two private educational institutions in the City of Poza Rica, Veracruz, about their sexual education. The study presents results obtained through the application of an instrument consisting of 29 questions focused on knowing the opinion of adolescents about their sexual education, contraceptive methods and teenage pregnancy. The objective of the research is to know the perspective of the students of two private and two public educational institutions on sexual education and teenage pregnancy, to establish proposals on a comprehensive Sexual Education.
\end{abstract}

Sex education, Teens, Social Work

Citación: PACHECO-ARENAS, Noelia, MERCADO-MOJICA, Alin Jannet y HERNÁDEZ-MAQUEDA, Martha Soledad. Estudio comparativo de opinión de adolescentes sobre Educación Sexual en instituciones públicas y privadas. Revista de Educación Básica. 2019. 3-8: 9-21.

\footnotetext{
*Correspondencia al Autor (Correo Electrónico: npacheco@uv.mx)

$\dagger$ Investigador contribuyendo como primer autor.
} 


\section{Introducción}

Según Rejón (2019) en el artículo "No hay educación sexual en México: expertos", del periódico la Jornada, en México "La educación sexual es absolutamente insuficiente y deficiente", dice la doctora Sandra Peniche Quintal, la primera mujer profesionista sexóloga en el sureste del país y directora de la clínica Servicios Humanitarios en Salud Sexual y Reproductiva A.C.,... la sexualidad es la base de la salud mental y social porque a partir de ahí nos construimos como personas y establecemos vínculos con los demás"...."no hay educación integral de la sexualidad en Yucatán y en el resto del país es una afirmación en la que coinciden especialistas en ginecología, sexología y mujeres entrevistadas.

En este orden de ideas, se presenta la educación sexual integral como un tema que aún requiere de transitar un amplio camino para lograr una formación sobre sexualidad, con mayor éxito en nuestra sociedad, que permita disminuir los problemas de embarazos no deseados, infecciones de transmisión sexual, violación de los derechos sexuales $\mathrm{y}$ reproductivos, entre otros.

Por otra parte, se plantea que desde el sector educativo el tema de la sexualidad se aborda únicamente desde el aspecto biológico, sin considerar las variables sociológicas e históricas de los grupos sociales, sin considerar además los aspectos médico, social, psicológico $\mathrm{y}$ emocional.

En el mismo artículo, Rejón et al. Menciona que el "director del Centro de Estudios Superiores en Sexualidad (Cessex), Frederick Santana Núñez, coincide, a grandes rasgos, con Peniche. Opina que no hay educación integral de la sexualidad porque no hay suficientes profesionales que la aborden de manera laica, desmitificante y científica. Él considera que desafortunadamente, docentes de todos los niveles siguen enseñando con bases sólo biológicas sin tomar en cuenta aspectos sociológicos e históricos. La sexualidad está conformada por muchas otras áreas: médica, cultural, social, psicológica, emocional”.
Por todo ello, abordar el tema de la educación sexual integral continua siendo un reto importante para el sector salud y desde la profesión de las/os Licenciado en Trabajo Social, también constituye un escenario vigente de investigación e intervención profesional, ya que los niños y jóvenes del siglo XXI presentan nuevas condiciones al tener accesibilidad ilimitada a medios de comunicación, que les brindan información sobre el tema de la sexualidad, sin embargo no cuentan con la capacitación o conocimientos sobre la formas de discernir la información fidedigna de la que no lo es, por lo que pueden caer en realizar prácticas sexuales de riesgo y aún más, promoverlas entre sus iguales.

Por lo que, surge el interés de realizar una investigación con el objetivo de conocer la perspectiva de los estudiantes de dos instituciones educativas privadas y dos públicas, sobre educación sexual y embarazo adolescente, para establecer propuestas sobre una Educación Sexual integral, trabajo desarrollado en el año 2018, en coordinación con las autoridades municipales de la ciudad de Poza Rica, Veracruz y el Consejo Estatal de Población (COESPO) del estado de Veracruz, en el marco de la Estrategia Nacional para la Prevención del Embarazo en Adolescentes (ENAPEA). Con el estudio se podrá analizar cuáles son las condiciones que diferencian los conocimientos que poseen las/os adolescentes sobre educación sexual, embarazo adolescente y métodos anticonceptivos, en función de la formación que reciben en instituciones públicas y privadas. Se estableció como hipótesis que a mayor nivel de acciones realizadas por las instituciones públicas o privadas sobre educación sexual, mayor será el nivel de conocimientos de los adolescentes sobre el tema.

La estructura del artículo se conformó por 5 secciones: 
Se da inicio al artículo con la Introducción que presenta un abordaje general del tema y centra la problemática, 1. Desarrollo que aborda el marco teórico que da sustento a la investigación con los temas de adolescencia y sexualidad, embarazo adolescente y métodos anticonceptivos; 2. Metodología que plantea el proceso de la investigación que se realizó de corte cuantitativo y de tipo correlacional, se utilizó la técnica de encuesta y el instrumento utilizado fue el cuestionario; 3. Resultados, esta sección presenta los datos más significativos obtenidos en la investigación que permiten comprobar la hipótesis; 4. Conclusiones sobre la percepción de educación sexual, embarazo adolescente y métodos anticonceptivos y finalmente 5. Propuestas generadas a partir de los resultados esperados.

\section{Desarrollo}

\section{Adolescencia y educación sexual}

Para el ser humano la sexualidad es una dimensión fundamental, ya que está íntimamente relacionada con la afectividad, la capacidad de amar y la aptitud para relacionarse con los demás. De acuerdo con la Organización Mundial de la Salud (OMS, 2018), la sexualidad humana se define como:

"Un aspecto central del ser humano, presente a lo largo de su vida. Abarca el sexo, las identidades y los roles de género, la orientación sexual, el erotismo, el placer, la intimidad y la reproducción. Se siente y se expresa a través de pensamientos, fantasías, deseos, creencias, actitudes, valores, comportamientos, prácticas, roles y relaciones. $\mathrm{Si}$ bien la sexualidad puede incluir todas estas dimensiones, no todas ellas se experimentan o expresan siempre. La sexualidad está influida por la interacción de factores biológicos, psicológicos, sociales, económicos, políticos, culturales, éticos, legales, históricos, religiosos y espirituales". (p. 3)
El ser humano presenta procesos de transición en cada etapa de su vida, específicamente en la adolescencia experimenta cambios físicos, psicológicos y sociales, que llevan a la aparición de los rasgos de la adultez, se vive una etapa de descubrimientos, de impulsos y de errores y aunque no todos los adolescentes se desarrollan al mismo tiempo y del mismo modo, hay factores comunes que los hacen a todos tan vulnerables como la propia etapa en la que viven, uno de los más importantes es el despertar de la sexualidad.

Hoy en día las/os adolescentes son mayormente sensibles a un mundo que les ofrece diariamente diversas motivaciones que estimulan el inicio temprano de la vida sexual, lo cual genera en muchos casos embarazos no deseados, la formación precoz de una familia, que generalmente se desarrolla en un contexto inestable y la presencia de enfermedades de trasmisión sexual; a pesar de que existe mucha información sobre sexualidad, no todos los adolescentes comprenden los conocimientos que necesitan saber para tomar decisiones en relación a su vida sexual.

Según Yañez (2015) en el artículo "Entrevista de mente y emociones", publicado en la revista de Salud y bienestar, establece que los adolescentes en ocasiones actúan por imitación, "les importa lo que opinen sus iguales y éstos de alguna manera ejercen una cierta presión sobre ellos", por lo que si sus amigos inician una vida sexual a temprana edad, incitará que ellos deseen iniciar su vida sexual, aunque es importante señalar que el comportamiento sexual de los adolescentes no sólo depende de la influencia de sus iguales sino de otros factores que cobran igual importancia. Por ejemplo, los cambios físicos, la erotización de la sociedad o los factores culturales y familiares y por último, la presión que puede ejercer en ellos la propia pareja”.

A pesar del avance en la sociedad y los cambios en la percepción de la pareja y del ideal romántico, los adolescentes siguen teniendo algunos mitos del amor, muchas mujeres inician sus primeras relaciones sexuales asociadas al sentimiento de amor y los varones más en relación a la sensación de placer. 
Si bien en la adolescencia se está físicamente preparado para el inicio de una vida sexual, no se tiene la madurez que implica compartir una relación sexual en la que están involucrados la salud, los pensamientos, los sentimientos y sensaciones, la comunicación y sobre todo el respeto.

Por ello, cobra vital importancia la educación sexual integral, en este sentido la UNESCO (2018, p.1) publicó en el mes de febrero en su sitio web, una edición de las Orientaciones técnicas internacionales sobre educación en sexualidad que promueve la educación sexual integral de calidad, así como como la igualdad de género, y empodera a niños y jóvenes para que lleven una vida sana, segura y productiva.

Si bien desde el sector educativo se imparte "Educación sexual" es importante fortalecer estrategias que permitan a las/os adolescentes tomar decisiones fundamentadas sobre su sexualidad, incorporando información de acuerdo a la edad, desarrollo humano, anatomía y embarazo, asimismo, incluir información sobre anticoncepción e infecciones de transmisión sexual (ITS), entre ellas el Virus de Inmunodeficiencia Humana (VIH), es necesario ir más allá de la información para fomentar la confianza y la mejora de las aptitudes de comunicación, abordar también las cuestiones sociales que rodean a la sexualidad y la reproducción, entre ellas las normas culturales, la vida familiar y las relaciones interpersonales.

La educación sexual ayuda y enseña a las personas a obtener la información, las herramientas de calidad y la motivación necesarias para tomar decisiones saludables sobre el sexo y la sexualidad, constituye una estrategia para el bienestar y calidad de vida de la población, donde las/os Trabajadoras/es Sociales deben promover que la educación sexual se integre al proceso formativo escolar desde las primeras etapas de la vida: la niñez y adolescencia, de manera transversal y permanente, integrando además procesos de capacitación a padres de familia, para que sean quienes refuercen la educación sexual en el contexto familiar, propiciando saberes teóricos - heurísticos y axiológicos sobre el tema.
En este contexto, se puede decir que debido a que durante la adolescencia el ejercicio responsable, respetuoso y equitativo de las relaciones interpersonales es fundamental para disminuir conductas sexuales riesgosas. En México, la Encuesta Nacional de Salud y Nutrición (Ensanut, 2012, p. 79) ha evidenciado que de las mujeres adolescentes con inicio de vida sexual $51.3 \%$ había estado alguna vez embarazada.

Aunque $90 \%$ de la población adolescente reportó conocer o haber escuchado hablar de algún método anticonceptivo, 15\% de los hombres y $33 \%$ de las mujeres no utilizaron método anticonceptivo alguno en su primera relación sexual, lo que evidencia la necesidad de la población adolescente de recibir una Educación Sexual Integral para que los adolescentes conozcan sus mecanismos de acción y efectividad, lo cual les permitirá utilizarla de manera sistemática y consciente en la toma de decisiones de su vida, evitando con ello enfermedades de transmisión sexual, embarazos no deseados, abortos, deserción escolar, incluso postergar o truncar su proyecto de vida profesional.

\section{Embarazo adolescente}

El embarazo en la adolescencia se ha convertido en un problema de salud pública y social, que afecta negativamente en la salud de las jóvenes mujeres y en la de sus hijos/as; el embarazo en esta etapa ya no es una situación exclusiva que se relaciona con grupos en condiciones socioeconómicas desventajosas y con bajos niveles educativos, pues en otros sectores, especialmente en áreas urbanas, la maternidad crece entre jovencitas con educación media o media superior.

Según la Organización Mundial de la Salud (OMS, 2018), un millón de niñas menores de 15 años y 16 millones de jóvenes de entre 15 y 19 años dan a luz cada año, aunado a 3 millones aproximadamente que se someten a abortos clandestinos y peligrosos. Las complicaciones durante el embarazo y el parto son la segunda causa de muerte entre las jóvenes en estos rangos de edad. 
Según el, periódico El País (26 de marzo, 2019) en el artículo "Embarazo adolescente en México: un problema alimentado por la inequidad" México tiene la mayor tasa de natalidad en mujeres adolescentes de todos los países miembros de la Organización para la Cooperación y Desarrollo Económico (OCDE): 62 embarazos por cada 1.000 son de niñas y adolescentes. Pese a que entre los años 2000 y 2006 se logró una reducción de más del $8 \%$, entre el 2007 y el 2012 el incremento llegó casi al 15\%. En 2017, dos de cada diez nacimientos en México eran de una mujer menor de 20 años.

Rodríguez, del Pilar, Aguilar, Ribeiro, Amaya, \& Henao (2019) señalan que el embarazo en la adolescencia es diferencial de acuerdo al nivel socioeconómico, siendo una constante que en los niveles socioeconómicos más bajos se presente con mayor frecuencia, de acuerdo con el último informe del Estado mundial de las madres que cada año realiza la ONG Save the Children, se evidencia la discrepancia en la prevalencia de uso de métodos anticonceptivos entre los estados más al sur del país, como Chiapas, con 35,5\%, y el resto, donde se estima su uso en un 59\%, dado que el acceso a los anticonceptivos entre adolescentes de 15 a 19 años de edad sexualmente activas no es parejo a lo largo del país, la diferencia más importante en cuanto a salud reproductiva tiene que ver principalmente con la inequidad en el acceso a los servicios de salud, la cual se encuentra claramente marcada por los estratos socioeconómicos.

Según el Grupo Estatal para la Prevención del Embarazo en Adolescentes de Veracruz (GEPEA, 2018) para el estado de Veracruz:

En 2017, en su informe de Alerta de Género por Agravio Comparados, la Comisión Nacional para Prevenir y Erradicar la Violencia contra las Mujeres (CONAVIM) de la Secretaría de Gobernación, en el tema de embarazos adolescentes ubicó a Veracruz a finales de 2016 y principios de 2017 en el segundo lugar nacional con el registro de $26 \mathrm{mil}$ embarazos, en mujeres con edad de 15 a 19 años.
Ese mismo año la dirección de salud pública de la Secretaría de Salud y Dirección de Servicios de Salud de Veracruz (SESVER) y el Consejo Estatal de Población (COESPO) confirmaron la información dada a conocer por la CONAVIM y destacaron que el estado de Veracruz ocupa el segundo lugar a nivel nacional en embarazos de mujeres adolescentes, pues de 26 mil embarazos registrados en el año 2016, el 91 por ciento era de mujeres de entre 15 a 19 años, y el 8 por ciento de estos fue protagonizado por niñas menores de 15 años. (p.4)

Ante todo este escenario, se puede decir que a pesar de que existen servicios de salud que buscan cubrir las necesidades de las/os adolescentes y a los diversos programas sobre educación sexual, ésta sigue siendo un reto para el sector salud y requiere una mayor integración con el sector educativo. En este sentido, es importante reconocer que los jóvenes no acuden a las instituciones de salud por métodos anticonceptivos, no obstante, quienes sí acuden a solicitar dichos métodos no reciben la orientación suficiente, por lo que surge la necesidad de diseñar políticas que permitan a los/as adolescentes obtener mayor información y uso correcto de los métodos anticonceptivos, que coadyuven a disminuir los embarazos a temprana edad.

\section{Métodos anticonceptivos}

Las tareas que deben afrontar los adolescentes en su proceso de maduración son múltiples, aceptar los cambios que experimentan sus cuerpos, asumir nuevas responsabilidades, atender al creciente deseo sexual que les empuja a relacionarse afectiva y sexualmente con otras personas, siendo el ejercicio de su sexualidad y el compromiso que esto conlleva para su salud reproductiva uno de los principales desafíos para evitar una gestación no deseada y de no contraer ITS.

Promover el uso de métodos anticonceptivos durante la adolescencia es controversial, dado que se considera que con ello se despierta el interés de las/os adolescentes por el inicio de una vida sexual, lo ideal sería que los adolescentes no mantuvieran la primera relación sexual coital hasta que estén psicológicamente preparados y tengan pleno conocimiento del uso adecuado de los métodos anticonceptivos. 
Sin embargo, cada vez los niño/as y adolescentes inician su vida sexual a más temprana edad y las evidencias de ello son contundentes.

Lo más importante es capacitar a las/os adolescentes sobre los diversos métodos anticonceptivos, analizando los valores, las condiciones biológicas, psicoafectivas y socioeconómicas que presentan. Es importante incluir los temas sobre métodos de emergencia, con una conciencia de que no deben ser de uso recurrente, porque su objetivo es prevenir un posible embarazo cuando se han mantenido relaciones sexuales sin protección, o en el caso de que ésta falle, haciendo énfasis en que estos métodos no previenen enfermedades de transmisión sexual.

Para ello es pertinente que desde la intervención de Trabajo Social en el ámbito educativo se realice la difusión de la Guía "Recomendaciones sobre prácticas seleccionadas para el uso de anticonceptivos" que tiene la OMS, la cual ofrece un asesoramiento sobre cómo usar el método anticonceptivo elegido de una forma segura y eficaz, haciendo hincapié en la necesidad prioritaria de asistir a los servicios de salud, con profesionales que les brinden orientación y asesoría especializada para evitar riesgos y complicaciones en su salud.

Esta misma guía plantea que los métodos anticonceptivos en la adolescencia se pueden clasificar en:

Recomendables/Preferentes: preservativo, anticoncepción hormonal combinada (oral, transdérmica y anillo vaginal), anticoncepción hormonal oral de solo progestágeno y dispositivo intrauterino (DIU).

Aceptables: anticoncepción hormonal de depósito (inyectables e implantes) y diafragma.

Poco aceptables: métodos naturales y esterilización quirúrgica.

De emergencia: anticoncepción poscoital.
Un factor determinante en el uso de métodos anticonceptivos en la población adolescente es la disposición y la facilidad de acceso a ellos, algunas de las limitantes pueden ser cuestiones socioculturales como son mitos, tradiciones (llegar virgen al matrimonio), vergüenza de solicitarlos cuando son mujeres solteras, desconocimiento del acceso gratuito de métodos anticonceptivos por instituciones de salud, entre otros.

En México la Ensanut (2012) refiere que $32.7 \%$ de las/os adolescentes mencionan haber obtenido los condones gratuitamente en el sistema de salud donde el Instituto de Seguridad y Servicios Sociales de los Trabajadores del Estado (ISSSTE) aparece como principal proveedor, por lo que es una población muy baja la que manifiesta tener el acceso gratuito, aunado a ello, el no hacer un uso adecuado de los mismos, plantea la posibilidad de presentar embarazos no deseados, así como ITS.

Por tal motivo, en México se ha puesto en marcha la Estrategia Nacional para la Prevención del Embarazo en Adolescentes (ENAPEA), en coordinación con Consejo Nacional de Población (CONAPO), con el objetivo de reducir el número de embarazos en adolescentes, a través de la cual se aborda, de manera actualizada contenidos sobre el tema de la sexualidad, además de favorecer una orientación sin prejuicios sobre la adolescencia, los derechos sexuales, la salud sexual y reproductiva.

\section{Metodología a desarrollar}

La investigación que se realizó fue de corte cuantitativo, Hernández, Fernández y Baptista (2014, p.4) refiere que "la metodología cuantitativa es aquella que elige una idea, que deriva hipótesis y variables; desarrolla un plan para probarlas; mide las variables, analiza las mediciones obtenidas y establece una serie de conclusiones respecto de la(s) hipótesis." Ya que pretende establecer un proceso de comprobación de hipótesis, analizar los resultados y determinar las condiciones que se permitan en los sujetos de estudio, llegando a establecer las conclusiones respecto a la opinión que tienen los jóvenes estudiantes. 
Es de tipo correlacional, como plantean Hernández Fernández y Baptista (2014, P. 81) Asocia variables a un patrón predecible para un grupo o población y tiene como finalidad conocer la relación o grado de asociación que exista entre dos o más conceptos, categorías o variables en un contexto en particular. La presente investigación pretende describir las diferencias de opinión que tienen las/os adolescentes sobre los conocimientos que poseen sobre educación sexual, considerando como variable las condiciones que tienen las instituciones públicas y privadas.

La población a estudiar se conformó por una muestra representativa conformada por 299 adolescentes, de los cuales 190 fueron de dos instituciones públicas (100 y 90) y 109 de dos instituciones privadas (58 y 51), de la ciudad de Poza Rica, Veracruz. Esta muestra representa el $48.53 \%$ de la población total, que conforman las instituciones participantes y que representan de manera proporcional a la población, según los grupos y secciones vigentes en el ciclo escolar.

El proceso de selección se realizó de manera aleatoria, considerando que fueran adolescentes del nivel de secundaria, inscritos en el ciclo escolar 2018- 2019, que asistieran a las instituciones el día de la aplicación de la encuesta y tuvieran interés en participar en la aplicación de la meta.

La técnica aplicada fue la encuesta, la cual consiste en recopilar información sobre una parte de la población denominada muestra, por ejemplo: datos generales, opiniones, sugerencias o respuestas que se proporcionen a preguntas formuladas sobre los diversos indicadores que se pretenden explorar a través de este medio. La información recogida podrá emplearse para un análisis cuantitativo con el fin de identificar y conocer la magnitud de los problemas que se suponen o se conocen en forma parcial e imprecisa. También puede utilizarse para un análisis de correlación para probar hipótesis descriptivas (Rojas, 2013, p. 221).

En la investigación la encuesta posibilitó obtener información precisa sobre las variables a estudiar para estar en condiciones de valorar y en su caso comprobar la hipótesis, a través de la opinión generada por las/os adolescentes participantes en la investigación.
El instrumento fue un cuestionario el cual estuvo conformado por 29 preguntas, en las cuales se abordan aspectos generales, conocimiento sobre educación sexual, métodos anticonceptivos y embarazo en la adolescencia. El instrumento se construyó a partir del Cuestionario de educación integral en sexualidad diseñado por el Consejo Estatal de Población (COESPO) del Estado de Veracruz, integrando 2 preguntas abiertas y 27 de opción múltiple. El procesamiento de la información se realizó utilizando el paquete estadístico para las ciencias sociales SPSS 25, desarrollado por IBM; es un sistema amplio y flexible de análisis estadístico y gestión de información que es capaz de trabajar con datos procedentes de distintos formatos. Para el procesamiento de información, se realizó la base de datos en Excel y posteriormente se exportaron al SPSS 25 para realizar el procesamiento de información en tablas y gráficos sobre cada variable, para realizar la comparación de resultados obtenidos en instituciones públicas, frente a los resultados obtenidos en instituciones privadas. Como etapa final del artículo se presentan las conclusiones y propuestas.

\section{Resultados}

El desarrollo de la investigación permitió establecer los siguientes resultados obtenidos del estudio comparativo realizado en dos instituciones educativas de nivel secundaria del sector público y dos del sector privado.

Edad: Los resultados obtenidos respecto a la edad son significativos ya que se pudo observar que en las instituciones privadas de las/os estudiantes tienen una edad de entre $14 \mathrm{y}$ 19 años, siendo significativo que $65.8 \%$ tienen una edad de entre 16 y 19 años, distribuidas principalmente en edad de 16 años $34.2 \%$ y 17 años con 23.7\%; en tanto que, en las instituciones públicas el rango de edad está ubicado entre los 11 y 15 años, lo cual representa la edad promedio para cursar la secundaria, esto indica que en las instituciones privadas los adolescentes tiene la oportunidad de cursar la secundaria aún cuando tienen una edad mayor a 15 años, ya que según la Secretaría de Educación pública (SEP) (Gobierno de México, 2017) "La secundaria es obligatoria y se proporciona en tres años a la población de 12 a 16 años que haya concluido la educación primaria. 
Las personas mayores de 16 años pueden estudiar en la secundaria para trabajadores o en la modalidad para adultos.”

Sexo de la población participante: Respecto a este rubro se encontró que en las instituciones privadas predominó la participación de mujeres con $97.4 \%$, en las instituciones públicas también predomino la población de mujeres con $52.9 \%$, lo que refleja que en las públicas se presentó una diferencia mínima de $5.8 \%$ en población inferior de hombres.

Definición de masturbación: Para abordar el nivel de conocimientos que poseen la/os estudiantes sobre el tema de sexualidad, se planteó la definición de masturbación, identificando que de los siguientes rubros, reportados se obtuvieron resultados de las instituciones privadas/ instituciones públicas de la siguiente forma:

Un acto para conocer mi cuerpo: 39.5 $\% / 32.1 \%$; Un acto que puede afectar mi salud física y mental: $50.0 \% / 23.5 \%$; Una forma de relacionarme con mi pareja: $2.6 \% / 0.5 \%$; Una forma dañina de sentir placer: $15.8 \% / 4.3 \%$; Un acto que me produce culpa: $2.6 \% / 0.5 \%$ y No sé: $13.2 \% / 39.0 \%$.

Se puede concluir que en las instituciones privadas tienen mayor precisión del concepto de masturbación, aunque en ambos tipos de institución, la población que conoce la definición es menor con una diferencia de $7.4 \%$ menor en las públicas, haciendo énfasis que predomina la población que desconoce o tiene una definición errónea del concepto este de $84.2 \%$ que desconoce en las privadas y $67.8 \%$ en escuelas públicas, considerando que en las instituciones privadas son de mayor edad y cuentan con departamento de apoyo psicopedagógico y orientación que favorece la realización de cursos y actividades sobre sexualidad.

Edad ideal para iniciar una vida sexual: Las/os adolescentes plantearon que la edad ideal para iniciar una vida sexual es preferentemente, en las privadas de 18 años con $26.3 \%$, en tanto que en las públicas consideraron que sería de 20 años representada por $25.1 \%$, ambos grupos de población consideran ideal iniciar una vida sexual después de la mayoría de edad, para tener un mayor nivel de madurez física y mental.

ISSN-2523-2452

ECORFAN $^{\circledR}$ Todos los derechos reservados
Has tenido al menos una relación sexual: Respecto al inicio de una vida sexual activa en la población investigada, se identificó que las/os adolescentes respondieron haber tenido al menos una relación sexual, en las instituciones privadas $31.6 \%$ refiere haber tenido ya alguna relación sexual, en tanto que en la instituciones públicas sólo el $2.7 \%$ contestó de manera afirmativa, presentando una diferencia de $28.9 \%$; sin embargo es significado identificar que en las instituciones públicas 92.5\% no respondió la pregunta, lo cual permite identificar que al ser una población con menor rango de edad (11-15) no tuvo la confianza de dar una respuesta al respecto, lo que no significa que no hayan tenido al menos una relación sexual en esta etapa de su vida.

Motivo más importante por el cual decidiste tener tu primera relación sexual: De la población que ha dado inicio a su vida sexual, refirió que el motivo por el cual decido tener su primera relación sexual, de las instituciones privadas $21.1 \%$ señaló que por deseo, $5.3 \%$ por amor y el mismo porcentaje por curiosidad, sólo $2.6 \%$ por presión del novio/a y un significativo $65.8 \%$ no respondió; en tanto que, los datos de las escuelas públicas refieren que 95.2\% No respondió, $3.7 \%$ fue por curiosidad y sólo $1.1 \%$ por deseo. En ambos casos el mayor porcentaje de población no respondió considerando que el tema de la sexualidad continúa siendo un tema muy hermético aun en el contexto del siglo XXI.

Motivo para decidir no tener tu primera relación sexual: De la población adolescente que respondió no haber tenido su primera relación sexual planteó los siguientes argumentos que sustentan su decisión, contestando en algunos casos más de una respuesta: en las instituciones privadas predomino con $18.4 \%$ No correr riesgos de un embarazo y $18.4 \%$ por sus valores y creencias; $\mathrm{y}$ en las instituciones privadas $52.4 \%$ quiere esperar a ser mayor y otro $52.4 \%$ por valores y creencias. Se puede concluir que tanto en las instituciones públicas y privadas los motivos para no iniciar una vida sexual son semejantes, por un lado, el temor a un embarazo a temprana edad lo cual refleja una percepción clara de las implicaciones del embarazo adolescente en su proyecto de vida, aunque hay una diferencia menor representada por $34 \%$ en las respuestas de la población de instituciones privadas. 
Conocimientos sobre los derechos sexuales y reproductivos: Respecto al nivel de conocimiento sobre los derechos sexuales y reproductivos las/os adolescentes, de las instituciones privadas que desconocen los derechos sexuales representa $81.6 \%$ y públicas $72.2 \%$, es significativo que en ambos sectores existen un amplio desconocimiento sobre el tema ya que únicamente el $18.4 \%$ y el $27.8 \%$ de las instituciones privada y públicas respectivamente manifestaron conocerlos, siendo mayor la población que tiene información sobre el tema de las instituciones públicas, sin embargo esto no garantiza que tengan una comprensión completa de los mismos y por tanto sean capaces de ejercerlos en su vida cotidiana.

Contagio de una infección de transmisión sexual: Respecto de haber tenido algún contagio por alguna infección de trasmisión sexual, la población que ha iniciado su vida sexual, de las escuelas privadas $2.6 \%$ frente a un $0.5 \%$ en las escuelas públicas dieron una respuesta afirmativa, lo cual refiere que al tener mayor conocimiento sobre educación sexual presentan menor riesgo de contraer alguna ITS.

Formas de transmisión de VIH/SIDA: De las respuestas obtenidas respecto al conocimiento que tiene la población estudiada sobre las formas de contraer el VIH/SIDA, pudiendo señalar más de una respuesta, se identificó que la mayor cantidad de porcentajes se ubica correctamente, en ambas instituciones obtuvieron los mayores porcentajes en las causas de contagio representadas respectivamente en las escuelas privadas/públicas, por: relaciones sexuales sin protección 100\%/91.4\%; por trasfusión de sangre $94.7 \% / 85.6 \%$ y por intercambio de fluidos 73.7\%/66.3\%; sin embargo cabe resaltar que dentro de las respuestas erróneas se destaca por compartir artículos de higiene personal 65.8\%/66.8\%; durante el embarazo 50\%/48.1\%; con un beso $23.7 \% / 29.4 \%$; por piquete de mosco $23.7 \% / 32.2 \%$ y con un abrazo $10.5 \%$ $119.8 \%$. Se puede concluir que aún existen mitos y conocimientos erróneos sobre las formas de contagio del VIH/SIDA a pesar de la información que se trasmite en las instituciones educativas e instituciones de salud.
Informes y Donación de Métodos Anticonceptivos: Respecto a los servicios que han recibido las/os adolescentes relacionados a su sexualidad, se identificó que los datos reportados por las instituciones privas / públicas son los siguientes: Los resultados obtenidos respecto haber recibido informes y donación de métodos anticonceptivos, se encontró que hay más participación en la población de instituciones públicas con $31.6 \%$, contra $23.7 \%$ en instituciones privadas, pero es aún más significativo que la población que nunca ha tenido acceso a información y métodos anticonceptivos es de $76.3 \%$ y $95.7 \%$ en las privadas y públicas respectivamente, por lo que es muy revelador que en la población no haya tenido información tanto en la propia institución educativa como de las instituciones del sector salud, haciendo énfasis en la necesidad de redoblar esfuerzos para que las/os adolescentes puedan acceder a estos servicios.

Los datos obtenidos referente a haber recibido asesoramiento o si se han realizado prueba de embarazo, se identificó que únicamente $2.6 \%$ de adolescentes de instituciones privadas los han recibido; en tanto que en las instituciones públicas es mayor la población que contestó de manera afirmativa $3.7 \%$.

Respecto a la afirmación de haber recibido educación sexual, en las privadas la mitad de la población considera que si la tenido acceso a ella, pero es más significativo que hay un incremento de $5.1 \%$ en las instituciones públicas, lo que no es congruente con el nivel de conocimiento que refieren sobre el tema de la sexualidad, es decir, que los conocimientos que tienen no son certeros.

Referente a material que han recibido sobre salud reproductiva son $18.4 \%$ y $13.9 \%$ en instituciones privadas y públicas respectivamente, lo que representan una mínima población que han tenido acceso a estos servicios del sector salud.

Es menor la población que han recibido información sobre planificación familiar, representado por $10.5 \%$ / $12.8 \%$ en instituciones privadas y públicas respectivamente 
Conocimiento de métodos anticonceptivos: Respecto al conocimiento de los métodos anticonceptivos, los adolescentes de las instituciones privadas/públicas señalaron tener mayor conocimiento de los métodos anticonceptivos de barrera con 94.7\%/85.0\% respectivamente; los anticonceptivos hormonales con $78.9 \% / 54.5 \%$; anticonceptivos naturales con $73.7 \%$ / 68.4\%; lo que refleja que tanto en las escuelas privadas como públicas conocen por lo menos algún método anticonceptivo; sin embargo cabe resaltar que $28 \% / 7.5 \%$ señalaron no conocer ningún método anticonceptivo pese a que las instituciones de salud y educativas promueven en conocimiento y uso de los métodos anticonceptivos para prevenir un embarazado no deseado o adquirir alguna ITS.

Uso de Métodos anticonceptivos: De la población adolescente participante que señalo haber tenido por lo menos una relación sexual, se les pregunto cuáles métodos anticonceptivos habían usado, teniendo la posibilidad de seleccionar más de una opción, obteniéndose que en las escuelas privadas/públicas las siguientes respuestas: Condón Masculino 28.9\% / 7.0\%; Píldora anticonceptiva 10.5\% / 1.6\%; Condón Femenino 7.9\% / 2.7\%; Inyectables 2.6\% / 1.6\%; Parche 2.6\% / 1.1\%; Espermicidas $\quad 2.6 \% / 0.5 \%$; Implante $0 \%$ / $0.5 \%$; por lo que el condón masculino es el método anticonceptivo más usado por los adolescentes, considerando que éste es uno de los métodos anticonceptivos de mayor promoción por los diferentes servicios de salud para evitar embarazos y la transmisión de infecciones sexuales, así como de fácil adquisición por su bajos costos o incluso obtenerse gratuitamente.

Consumo o ayuda para tomar píldora de emergencia: Aunque los métodos de anticoncepción de emergencia no sean métodos anticonceptivos como tal, porque su objetivo es prevenir un posible embarazo cuando se han mantenido relaciones sexuales sin protección. los resultados en instituciones públicas/privadas sobre el consumo o ayuda para usar la píldora de emergencia fue de 23.3\%/11.2\% respectivamente. Cabe señalar que importante que las/os adolescentes identifiquen que el uso de la píldora de emergencia no debe ser de manera regular, sino en ocasiones de riesgo y bajo supervisión médica.
Interrupción del embarazo: En el mundo millones de mujeres arriesgan sus vidas y su salud para poner fin a embarazos no deseados. $\mathrm{Al}$ preguntar a las adolescentes si se practicarían una interrupción del embarazo los estudiantes de escuelas privadas señalaron que Sí con $2.6 \%$, que No con $97.4 \%$ mientras que en las escuelas públicas $5.3 \%$ mencionaron que Sí se interrumpirían un embarazo, $91.4 \%$ que No lo haría y $3.2 \%$ no contestó; por lo que se puede identificar que en ambas poblaciones es mayor el porcentaje de adolescentes que señala No interrumpir un embarazo, lo cual es positivo dado que el aborto en la adolescencia constituye un elemento fundamental que afecta la salud reproductiva de este sector de la sociedad. Si bien es cierto que se ha logrado disminuir la mortalidad relacionada con este procedimiento, las complicaciones que se presentan aún siguen cobrando muchas muertes es mujeres adolescentes.

Apoyaría en la interrupción del embarazo: Por otro lado, al cuestionar a los adolescentes si apoyarían a una mujer a interrumpir un embarazo, los estudiantes de escuelas privadas señalaron $50 \%$ que Sí ayudarían y $50 \%$ que No lo harían, mientras que en las escuelas públicas $26.2 \%$ señaló que si apoyaría a una mujer a interrumpir un embarazo, mientras que el $71.7 \%$ señaló que No y $2.1 \%$ no contestó; por lo que se puede identificar que es mayor el porcentaje de adolescentes de escuelas privadas que si apoyarían una interrupción del embarazo.

\section{Conclusiones}

Hoy en día las/os adolescentes inician una vida sexual cada vez a más temprana edad, sin contar con los conocimientos necesarios para realizar una toma de decisiones asertiva, que favorezca su proyecto de vida. Si se considera que existen factores como la cultura, los tabúes, la escasa educación sexual completa o integral, la excesiva información errónea de los medios de comunicación digitales que están al alcance de la mano, entonces las/os adolescentes presentan mayores dificultades para adquirir un adecuado conocimiento sobre la salud sexual y reproductiva. 
En este contexto, las instituciones educativas del nivel secundaria y los profesionales de Trabajo Social asumen un gran reto, generar proceso articulados con las instituciones del sector salud para hacer llegar información fidedigna y accesible a las/os jóvenes adolescentes y a sus familias para avanzar hacia el cambio de paradigmas sobre la educación sexual integral que debe promover una vida libre, consciente responsable sobre la sexualidad de las/os adolescentes, esto se podrá lograr en la medida que existan datos específicos de las condiciones que presentan los jóvenes en la actualidad.

Así el resultado de la investigación realizada por Trabajadoras sociales sobre el tema "Estudio comparativo de opinión de adolescentes sobre Educación Sexual en instituciones públicas y privadas", permitió despejar la interrogante ¿Cuáles son las condiciones que diferencian los conocimientos que poseen las/os adolescentes sobre educación sexual, embarazo adolescente y métodos anticonceptivos, en función de las condiciones que presentan los estudiantes tanto de instituciones públicas, como privadas?

Al respecto se pudo identificar que las condiciones que presentan las instituciones privadas para apoyar las actividades sobre educación sexual con las/os adolescentes es que cuentan con un departamento psicopedagógico que brinda orientación y asesoría sobre el tema, sin embargo las/os adolescentes de las instituciones públicas refirieron respuestas más acertadas sobre los conceptos planteados como referente de la educación sexual recibida, lo cual indica que en conocimientos son más precisos los de las dos instituciones públicas participantes; porque las instituciones públicas tienen mayor vinculación con el sector salud para promover temas de educación sexual.
Respecto al alcance del objetivo de la investigación, se puede concluir que se logró conocer la perspectiva de los estudiantes de dos instituciones educativas privadas y dos públicas sobre educación sexual y embarazo adolescente, para establecer propuestas sobre una Educación Sexual integral, ya que se obtuvieron respuestas que permitieron valorar los saberes que poseen los estudiantes de dos instituciones privadas y dos instituciones públicas, que se encuentran en la etapa de vida de la adolescencia entre 11 y 19 años de edad, siendo los de edad mayor quienes asisten a instituciones privadas ya que sus edades fluctúan entre 14 y 19 años que representan estudiantes con una edad mayor del promedio permitido por la SEP para cursar el nivel de secundaria; en tanto que, en las instituciones públicas fue de entre 11 y 15 años, lo que representa la edad establecida para cursar el nivel de secundaria, ambos grupos de estudiantes, participaron en las encuestas que permitieron conocer la percepción que tienen sobre la educación sexual y el embarazo adolescente. Con los resultados se plantearon 5 propuestas para impulsar la educación sexual integral desde el ámbito educativo en el nivel de secundaria.

Los resultados obtenidos permitieron corroborar la hipótesis planteada "a mayor nivel de acciones realizadas por las instituciones públicas o privadas sobre educación sexual, mayor será el nivel de conocimientos de los adolescentes sobre el tema", como se puede establecer a continuación: Con los datos obtenidos en la investigación se puede concluir que las instituciones educativas de nivel secundaria realizan actividades como cursos, talleres, asesorías, charlas informativas y canalizaciones sobre el tema de sexualidad; en los resultados se pudo constatar que en las privadas existe un mayor número de charlas informativas representadas con $33.33 \%$, así como asesorías y canalizaciones en un mismo porcentaje, representado por $22.22 \%$; en tanto que, las instituciones públicas presentan mayor número de asesorías con $41.67 \%$, seguidas de canalizaciones con $25 \%$ y charlas informativas con $16.67 \%$, lo que significa que ambas instituciones consideran importante la formación de los estudiantes sobre educación sexual, sin embargo por el tamaño de la población estudiantil y por los vínculos con el sector salud, se ve claramente más incrementadas las acciones en las instituciones públicas.

PACHECO-ARENAS, Noelia, MERCADO-MOJICA, Alin Jannet y HERNÁDEZ-MAQUEDA, Martha Soledad. Estudio comparativo de opinión de adolescentes sobre Educación Sexual en instituciones públicas y privadas. Revista de Educación Básica. 2019 
Respecto al nivel de conocimientos que poseen las/os adolescentes sobre educación sexual, métodos anticonceptivos y embarazo adolescente, en el estudio comparativo realizado se puede concluir que:

Los estudiantes de las escuelas privadas tienen mayor precisión del concepto de masturbación, sobre los estudiantes de escuelas públicas, con una diferencia de $7.4 \%$.

Los estudiantes de escuelas públicas en un porcentaje mayor en $1.2 \%$ en relación a los de escuelas privadas señalan como edad ideal para el inicio de una vida sexual 20 años, reflejándose que a mayor edad pueden tener mayor madures para asimilar su sexualidad y las responsabilidades que esta implica.

Los estudiantes de escuelas públicas tienen mayor conocimiento en un $9.4 \%$, que los de instituciones privadas, sobre los Derechos sexuales y reproductivos, aunque es importante señalar que no se especifica si éstos los aplican a su vida cotidiana.

Respecto al conocimiento sobre las formas transmisión del VIH/SIDA los estudiantes de escuelas privadas mostraron un conocimiento superior en $8.6 \%$ respecto a las formas verdaderas, identificando con claridad que a través de tener relaciones sexuales sin protección, transfusiones sanguíneas e intercambio de fluidos se puede trasmitir el virus.

$>$ A pesar de la información que se trasmite por diversos medios se identifica que aún existen conocimientos erróneos sobre las formas de adquirir el VIH/SIDA, siendo los estudiantes de escuelas privadas quienes manifestaron con un porcentaje mayor a los de las escuelas públicas.

Existe un diferencia mayor en un $1.0 \%$ respecto a que el VIH/SIDA se puede transmitir por compartir artículos de higiene personal, $8.5 \%$ Por piquete de mosco y Por un beso5.7\%, en tanto que solo es superior la diferencia representada por $1.9 \%$ en las instituciones privadas que se transmite durante el embarazo.
En general se puede decir que las/os adolescentes de instituciones públicas investigadas presentan un mayor índice de conocimientos erróneos sobre las formas de adquisición del VIH/SIDA.

Respecto a los servicios de salud sexual y reproductiva que reciben los adolescentes, los estudiantes de escuelas púbicas señalaron tener mayor información y recibir la donación de métodos anticonceptivos sobre los estudiantes de escuelas privada, representada con una diferencia de $7.9 \%$.

Las/os adolescentes de escuelas públicas señalaron en mayor porcentaje de $1.1 . \%$ de diferencia sobre las/os adolescentes de escuelas privadas haber recibió asesoría sobre la salud sexual y reproductiva, así como de pruebas de embarazo.

Existe una mayor proporción de 5.1\% en las respuestas de adolescentes de públicas respecto a haber recibió educación sexual de los servicios de salud, dado que las instituciones públicas mantienen mayor vinculación con los servicios de salud gubernamental.

Los adolescentes de escuelas públicas señalaron en un $2.3 \%$ de diferencia sobre los estudiantes de escuelas privadas, haber recibió información sobre planificación familiar por los servicios de salud. Lo que refleja mayor intervención de las instituciones de salud gubernamentales en las instituciones públicas.

Los adolescentes de escuelas privadas señalaron en mayor porcentaje, representado por $10.3 \%$ en promedio, conocer los métodos anticonceptivos como son los de barrera, hormonales, naturales.

En el uso de los métodos anticonceptivos se obtuvo una prevalencia mayor de $38.01 \%$ en las instituciones privadas frente a la pública, los métodos mayormente usados son Condón Masculino, píldora anticonceptiva y condón femenino.

Existe una diferencia superior de $2.7 \%$ de opinión de las/os adolescentes de instituciones públicas sobre la afirmación de considerar la posibilidad de realizarse una interrupción del embarazo. 
Los motivos por los cuales los adolescentes de instituciones públicas que han decidido no tener relaciones sexuales, se identifica una diferencia superior en los siguientes rubros: Quiero esperar a ser mayor con una diferencia de $39.2 \%$ y por sus valores y creencias con una diferencia de $34.0 \%$, mientras que en las intuiciones privadas solo fue superior en la respuesta No quiero correr el riesgo de un embarazo con una diferencia de $0.2 \%$. Lo que manifiesta que no se sienten preparados para afrontar la responsabilidad de ser padres a temprana edad.

Respecto a la posibilidad de apoyar a alguna mujer a interrumpir su embarazo, los adolescentes de escuelas privadas señalaron en mayor porcentaje representado por un $23.8 \%$ sobre las públicas, que sí apoyaría la interrupción del mismo.

\section{Propuestas}

$\checkmark$ Realizar un programa institucional que integre la participación de alumnos y padres de familia sobre el manejo de la educación sexual en el ámbito familiar.

$\checkmark$ Las instituciones privadas y públicas establezcan estrategias de vinculación con instituciones del sector salud y gubernamentales, para brindar orientación, asesoría y canalizaciones sobre temas relacionados a la educación sexual y el embarazo adolescente.

$\checkmark$ Crear programas institucionales que favorezcan la participación creativa e innovadora de los estudiantes en la formación de una educación sexual integral que les permita tomar decisiones más asertivas en su vida cotidiana y en sus relaciones interpersonales.

Articular un equipo de profesionales integrado por un Licenciado/a en Trabajo Social, un psicólogo, un médico y de ser posible contar con la asesoría de un sexólogo que impulse la educación sexual objetiva y científica.

$\checkmark$ Difusión en el ámbito educativo de la Guía "Recomendaciones sobre prácticas seleccionadas para el uso de anticonceptivos" emitido por la OMS

\section{Referencias}

Hernández, Fernández y Baptista (2014) Metodología de la investigación, McGRawHill. México (pág.4).

El país. (Marzo de 2019). Embarazo adolescente en México: un problema alimentado por la inequidad. Recuperado de https://elpais.com/elpais/2019/03/14/planeta_fut uro/1552580422_784534.html

Instituto Nacional de Salud Pública. (2012) Encuesta Nacional de Salud y Nutrición 2012.Recuperado:https://ensanut.insp.mx/infor mes/ENSANUT2012ResultadosNacionales.pdf

Rejón (22 de julio, 2019). No hay educación sexual en México: expertos. Jornada, Recuperado de https://www.lajornadamaya.mx/2019-0722/No-hay-educacion-sexual-integral-enMexico--expertos

Rodríguez, S. P. O., del Pilar Niño, B. A., Aguilar, S. S., Ribeiro, P. M., Amaya, C. M., \& Henao, L. S. (2019). Caracterización de adolescentes gestantes escolarizadas en instituciones educativas públicas de Bucaramanga, Colombia. Revista Cuidarte, 10(1), 6 .

Rojas, R. (2013). Guía para realizar investigaciones sociales. México. Editorial Plaza y Valdés.

OMS (2018a). Recomendaciones sobre prácticas seleccionadas para el uso de anticonceptivos, tercera edición. Recuperado de:

https://apps.who.int/iris/bitstream/handle/10665 /259814/9789243565408-

spa.pdf;jsessionid=AFF69D892AB1D6D4C430 832D0ED9B21E? sequence $=1$

OMS (2018b). La salud sexual y su relación con la salud reproductiva: un enfoque operativo. Recuperado de https://apps.who.int/iris/bitstream/handle/10665 /274656/9789243512884-spa.pdf

Grupo estatal para la prevención del embarazo en adolescentes de Veracruz. INFORME 2018. Recuperado de https://www.gob.mx/cms/uploads/attachment/fi le/461772/Informe2018_GEPEA_Veracruz.pdf 